\title{
ANALYSIS OF AN ARBITRARY PROFILE REFLECTOR ANTENNA SYSTEM HAVING RESISTIVE TYPE SURFACE - E-POLARIZATION CASE
}

\author{
Taner Oğuzer ${ }^{1}$, Ayhan Altintas² and A.I. Nosich ${ }^{3}$ \\ ${ }^{1}$ Department of Electrical and Electronics Eng. Dokuz Eylül University \\ Tinaztepe Campus 35160 Buca İzmir-TURKEY \\ ${ }^{2}$ Department of Electrical and Electronics Eng., Bilkent University, Bilkent Ankara-TURKEY \\ ${ }^{3}$ Institute of Radio-Physics and Electronics, National Academy of Sciences, Kharkov 61085, Ukraine
}

\begin{abstract}
The regularized solution is performed for arbitrary shape conic section profile geometry. In this case the reflector surface is taken as made up of resistive type material. The problem is formulated depending on the circular $2 \pi$ periodicity and then Fourier series coefficients of the surface current density are obtained. The resultant matrix equation is in the regularized form. Then the various numerical results are obtained for different eccentricity factor of the conic section and the resisitvity of the reflector surface.
\end{abstract}

\section{INTRODUCTION}

Reflector antenna system has the important part of the modern communication systems. Even though it is 3D in reality, the $2 \mathrm{D}$ equivalent can be though as the benchmark for the real reflector antenna system and it is called a canonical problem. The simulation of the reflector antennas have the main importance for the engineering point of view. Some analytical studies were performed in [1] for the circular perfectly conducting strip excited by the plane wave. In these studies some asymptotic expressions are obtained by enforcing high frequency approximation. Also the purely numerical techniques can be applied to this reflector antenna problem by the method of moments for electrically small and medium size geometries but the accuracy is not guaranteed and depends on the application. But in the real application quite large dishes are used and they can be simulated by high frequency asymptotic methods like GTD,UTD,PO or PTD. But the asymptotic technique does not produce single solution for all directions. It shows some caustics and transition region problems.

Another alternative for the analysis is method of the analytical regularization (MAR) technique [2]. Here some part of the operator is inverted analytically and the remaining part of the operator is solved numerically. Various problems are solved by this MAR technique. MAR technique is also applied to circular 2D reflector antenna problem in [3] for both polarizations. Then in [4] and [5], the arbitrary conic section profile reflector surface excited by a complex point source feed is analyzed and the resultant equations are in regularized form. So the high accuracy and convergence is obtained.

Here the same arbitrary conic section profile geometry is considered but in this case it is assumed to be a resistive reflector surface. Similar study was performed in [6] for the circularly curved strip case. But here in this case the reflector surface is taken as arbitrary profile. One can solve this problem geometry accurately by using certain method of analytical regularization (MAR). The main property of the geometry is its periodicity with $2 \pi$. Then all parameters can be expanded to the Fourier series and then the boundary condition is applied. The final equation is piece wise nature and then by using Fourier inversion procedure the coefficients of the surface current density are found and the surface curvature and resistivity are included into the solution. Then various numerical datas are obtained for the different reflector antenna geometries.

2. FORMULATION: Cross section of the problem geometry of infinitely thin resistively curved screen symmetrically illuminated by a directive feed is shown in Figure 1. As shown in Figure 1, it is a 2-D contour with an elliptic(or hyperbolic)-arc profile with the feed in a geometrical focus. The origin of the used coordinate system is taken just in this focus, and the point $\mathrm{O}_{\mathrm{e}}$ (or $\mathrm{O}_{\mathrm{h}}$ ) represents the symmetry center of the ellipse(or hyperbola). The reflector surface which is elliptic or hyperbolic one is completed to a closed one with a circle. The feed of this system is considered as complex source which produces a beam towards the reflector surface. The real position of the feed is the origin of the geometry. The resistivity of reflector surface is taken uniform and it is given an $\mathrm{R}=\mathrm{R}_{\mathrm{o}} \mathrm{Z}$ where $\mathrm{Z}$ is the free space intrinsic impedance.

When building the solution, an open arc of this generalized curve is completed to the closed contour $\mathrm{C}$ by a circle having its origin on the $\mathrm{x}$-axis. Its radius is chosen in such a way that at the connection points the 
curvatures of the arc and the circle are matched. Another condition for the solvability of the problem is that the branch cut associated with the CSP in the real space must not cross the strip contour M.

The requirements for the rigorous formulation of the considered boundary-value problem can be stated as the satisfaction of the Helmholtz equation, Sommerfeld radiation condition at far field, the resistive boundary condition on the reflector surface and the edge conditions at its endpoints. The basic EFIE can be obtained by applying resistive surface boundary condition valid in the E-polarization case.

$$
R_{0} Z J_{z}(\theta)=j k Z\left(E_{z}^{i n c}(\theta)+\int_{L=M U S} J_{z}\left(\theta^{\prime}\right) G\left(\vec{r}-\vec{r}^{\prime}\right) l\left(\theta^{\prime}\right) d \theta^{\prime}\right)
$$

Where $E_{z}^{i n c}=(i / 4) H_{0}^{(1)}\left(k\left|\vec{r}(\theta)-\vec{r}_{s}\right|\right)$ and then this incident field can be expanded into Fourier series with the argument of $b_{n}$ 's. Then the Free space 2D Green's function is $G=(i / 4) H_{0}^{(1)}\left(k\left|\vec{r}(\theta)-\vec{r}^{\prime}\left(\theta^{\prime}\right)\right|\right)$. Then the Green's function is to be expanded in terms of the double Fourier series. However this is not enough to regularize the problem, so a new function is introduce as follows:

$$
H\left(\theta, \theta^{\prime}\right)=H_{0}^{(1)}\left(k\left|\vec{r}(\theta)-\vec{r}^{\prime}\left(\theta^{\prime}\right)\right|\right)-H_{0}^{(1)}\left(2 k a_{s} \sin \frac{\left|\theta-\theta^{\prime}\right|}{2}\right)=\sum_{m, n=-\infty} g_{n m} e^{i n \theta} e^{i m \theta^{\prime}}
$$

The $\mathrm{H}$ function is chosen in such a way that $\mathrm{g}_{\mathrm{nm}}$ coefficients satisfy that [ 4]

$$
\sum_{m, n=-\infty}^{\infty}\left(|n|^{2}+1\right)\left(|m|^{2}+1\right)\left|g_{n m}\right|^{2}<\infty
$$

The Fourier series expansion of the $X\left(\theta^{\prime}\right)=J_{z}\left(\theta^{\prime}\right) l\left(\theta^{\prime}\right)=(2 /(i \pi)) \sum_{n=-\infty}^{\infty} x_{n} e^{i n \theta}$ is substituted into EFIE then after modifications the following regularized matrix equation is obtained.

$$
x_{m}+\left(\frac{k \pi}{2 R_{0}}\right) \sum_{n} x_{n} \sum_{p} Q(p-m) g_{p(-n)}+\left(\frac{k \pi}{2 R_{0}}\right) \sum_{n} x_{n} Q(n-m) J_{n} H_{n}^{(1)}(k a)=\frac{-i k \pi}{8 R_{0}} \sum_{u} b_{u} Q(u-m)
$$

Where Q function is defined as $Q_{n m}=Q(n-m)=\frac{1}{2 \pi} \int_{-\theta_{a p}}^{\theta_{a p}} l(\theta) e^{i(n-m) \theta} d \theta$.

3. NUMERICAL RESULTS: Various numerical results as the radiation patterns are obtained for the different reflector surface geometries and for the various surface resistivity. The results are compatible with the ones obtained in [6] for small aperture angle. Also the higher directivity and lower side lobes are obtained for the parabolic reflector case i.e. the $\mathrm{e}=1$, higher side lobe levels are obtained for hyperbola and ellipse cases.

4- CONCLUSION: The previous studies performed in literature is followed and so this time arbitrary profile resistive surface type reflector antenna system is formulated and the numerical results are obtained.

5 - ACKNOWLEDGEMENT: This work has been supported in part by the TUBITAK-NASU joint grant no. EEE AG-103E037.

\section{REFERENCES:}

[1] M. Idemen and A. Büyükaksoy, " High frequency surface currents induced on a perfectly conducting cylindrical reflector” IEEE Trans. On AP AP-32 1984, 501-507

[2] A.I. Nosich,"MAR in the wave scattering and eigenvalue problems: Foundations and review of solutions" IEEE AP magazine 42 1999, 34-39.

[3] T.Oğuzer, A.Altintas and A.I. Nosich, "Accurate simulation of the reflector antenna by complex source-dual series approach" IEEE Trans. On AP-43 1995, 793-802.

[4] Taner Oğuzer, Alexander I. Nosich and Ayhan Altıntaş "E-Polarized Beam Scattering by an Open Cylindrical PEC Strip having arbitrary Conical-Section Profile" Microwave Optical Technology Letters vol.31 no:6 pp 480-484 2001. 
[5] Taner Oğuzer, Alexander I. Nosich and Ayhan Altıntaş, "Analysis of an Arbitrary Profile Cylindrical Reflector Antenna, H-Polarization Case" accepted to the IEEE Transaction Antennas and Propagation for possible publication.

[6] Alexander I. Nosich, Vladimir B. Yurchenko and Ayhan Altintas, "Numerically exact analysis of a twodimensional variable resistvity reflector fed by a complex-point source" IEEE Trans. On AP-45 no:11 november 1997.

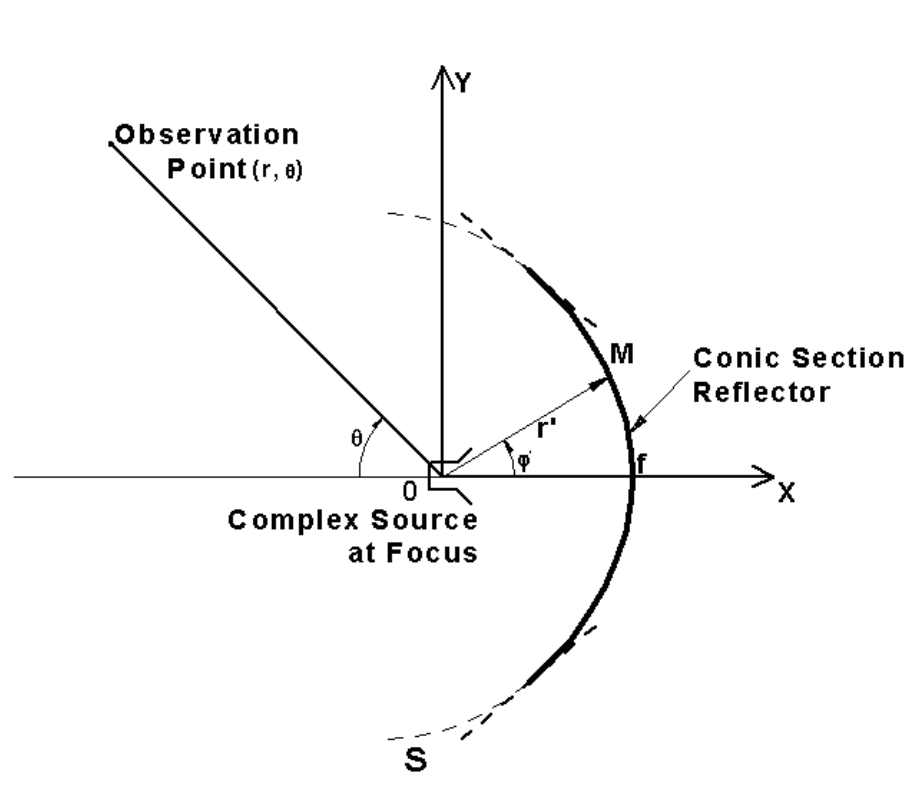

Fig. 1. The geometry of the problem
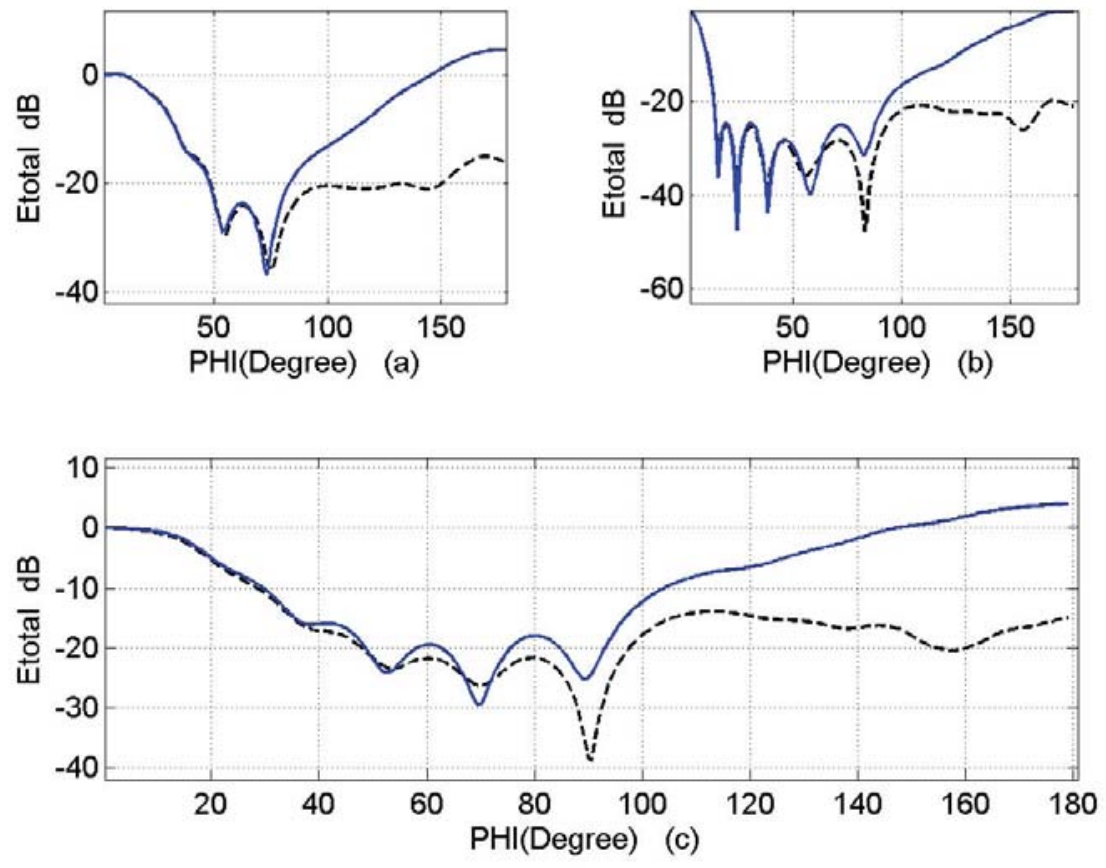

Fig. 2. Normalized radiation patterns for different eccentricity factors:

(a) $\mathrm{e}=0.25$, (b) $\mathrm{e}=1$ (c) $\mathrm{e}=4$. Also dashed line $\mathrm{R}=0.1 \mathrm{Z}$ solid line $\mathrm{R}=\mathrm{Z}$. 